\title{
Case-Based Reasoning to Classify Endodontic Retreatments
}

\author{
Livia Campo, Vicente Vera, Enrique Garcia, Juan F. De Paz, \\ and Juan M. Corchado
}

\begin{abstract}
Within the field of odontology, an analysis of the probability of success of endodontic retreatment facilitates the diagnostic and decision-making process of medical personnel. This study presents a case-based reasoning system that predicts the probability of success and failure of retreatments to avoid extraction. Different classifiers were applied during the reuse phase of the case-based reasoning process. The system was tested on a set of patients who received retreatments, and a set of variables considered to be of particular interest, were selected.
\end{abstract}

Keywords: case-based reasoning, classification, odontology.

\section{Introduction}

Predictive systems in medicine are relevant for determining the probability of success or failure of specific treatments. Their use currently extends to include various fields such as the study of cancer, or other fields such as odontology[1][2][3]. The decisions made by odontologists have been traditionally based on past experience of previous treatment cases, whereby experience in itself has been a necessary factor in the decision making process. There are normally too many variables to consider, which has in fact resulted in the high failure rate of retreatments.

Livia Campo $\cdot$ Vicente Vera $\cdot$ Enrique Garcia

Department of Estomatology II, Complutense University of Madrid

Plaza Ramón y Cajal, s/n 28040, Madrid, Spain

e-mail: lcampo@estumail.ucm.es, viventevera@odon.ucm.es,

aegarcialodon.ucm.es

Juan F. De Paz · Juan M. Corchado

Department of Computer Science and Automation, University of Salamanca

Plaza de la Merced, s/n, 37008, Salamanca, Spain

e-mail: $\{$ fcofds, corchado\} @usal.es

M.P. Rocha et al. (Eds.): 6th International Conference on PACBB, AISC 154, pp. 79-86. springerlink.com

(C) Springer-Verlag Berlin Heidelberg 2012 
Consequently, it has become necessary to create a system that facilitates the decision making process of odontologists, and results in the decisions that minimize the failure of endodontic treatments and retreatments.

Endodontics makes up 20\% of all treatments performed in dental clinics and has a $90 \%$ rate of success. The remaining $10 \%$ includes endodontic treatments that were unsuccessful to a greater or lesser degree, of which $40 \%$ are the result of root crown fractures, which in turn represent $5 \%$ of all dental fractures. The bacterial recolonization of the root canal and the subsequent appearance of radiological symptoms represent $15 \%$ of endodontic failure [8] [9] [10]. Many different alternative methods for analyzing data in odontology have already been investigated. The techniques applied in these fields are usually limited to the study of variables. A set of variables of interest is determined, followed by statistical tests and graphical representations of data to extract the relevant variables. Statistical analysis is limited to the application of specific tests such as chi square [12], MannWhitney [18] or Kruskal-Wallis [11]. These tests identify which variables present different characteristics in different groups; the value of the variables can subsequently be taken into consideration for the final classification. Nevertheless, it is necessary to create a process that can combine all the information gathered in order to perform a final classification. Previous Works in the field of bioinformatics CBR (Case-Based Reasoning) systems have been successfully applied to predict leukemia. This study proposes a reasoning system to predict the success of retreatments. A set of variables are recovered for a group of patients. This data set is used to generate a CBR system that incorporates different classification techniques during the reuse phase, in order to generate a classification for a new element. Traditional statistical techniques are applied during the revision phase to facilitate the interpretation of the results by selecting the variables that present different characteristics from those in the groups of individuals.

This article is divided as follows: section two describes the multi-agent systems and planning mechanisms used for assigning dynamic tasks; section three presents the proposed model; section four describe the results obtained and the conclusions respectively.

\section{Prediction System}

The use of predictive techniques in medicine and especially in the field of odontology has been studied since the late 80 s, having primarily used the statistical analysis of clinical data.

In 2001 Chungal N.M. published data related to a study of teeth extracted after unsuccessful endodontic treatments at the University of Connecticut School of Dental Medicine. The patients included in this study were treated between 1988 and 1992 in the graduate program and had experienced unsuccessful endodontic treatment within the previous four years. Variables were taken from both the clinical trial and x-rays taken at the time of the endodontic treatment. The data obtained in this case were studied with contingency tables and the chi-squared test. 
The risk factors were compared using t-tests for independent groups, or with nonparametric tests (Mann-Whitney or Kruskal-Wallis) [1].

Using the same characteristics, Givol, N. published the results in 2001 of his study performed in patients from private clinics in Israel. In this case, all the possible clinical variables prior and subsequent to the endodontic treatment were fathered from 5217 patients treated between 1992 and 2008. The data were also studied using statistical tests: chi-squared [2].

In July of 2011, Song, M. presented the data relative to a study performed on patients from the Department of Conservative Dentistry at the Dental College of Yonsei University, Seoul, Korea between August 2004 and December 2008. Included in this study were patients who had undergone unsuccessful endodontic treatment and were in need of periapical surgery. Song considered clinical and $\mathrm{x}$ ray data from prior to the treatment, demographic data, and data subsequent to the failed treatment. To analyze the factors that could predict the endodontic failure, he applied a chi-squared statistical study [3].

Of the previously cited studies, none used artificial intelligence or case base reasoning; nor did any use predictive tools other than the application of statistical studies to analyze risk factors. The use of this type of system offers, therefore, a wide area of study within the field of odontology and in particular with the prediction of unsuccessful endodontic treatments.

\section{Proposed Reasoning System}

The purpose of CBR is to solve new problems by adapting solutions that have been used to solve similar problems in the past [4]. The primary concept when working with CBRs is the concept of case. A case can be defined as a past experience, and is composed of three elements: a problem description which describes the initial problem, a solution which provides the sequence of actions carried out in order to solve the problem, and the final state which describes the state achieved once the solution was applied. A CBR manages cases (past experiences) to solve new problems. The way cases are managed is known as the CBR cycle, and consists of four sequential steps which are recalled every time a problem needs to be solved: retrieve, reuse, revise and retain. Each of the steps of the CBR life cycle requires a model or method in order to perform its mission. The algorithms selected for the retrieval of cases should be able to search the case base and select the problem and corresponding solution most similar to the new situation. Once the most important variables have been retrieved, the reuse phase begins, in which the solutions for the retrieved cases are adapted and a new solution is generated. The revise phase consists of an expert revision for the proposed solution. Finally, the retain phase allows the system to learn from the experiences obtained in the three previous phases, consequently updating the cases memory.

During the recovery phase, existing cases in which a retreatment was performed are selected from the case memory. This eliminates all cases that involve only an initial treatment. 
During the reuse phase, previously retrieved cases are selected and an associated classifier is built. In this case, the technique selected to carry out the classification phase corresponds to a Bayesian network. The new case is then introduced and classified according to the classifier built in this phase.

The Bayesian networks are constructed by following the Friedman-Goldsmidtz [5] algorithm. Having two different classes, two Bayesian networks will be generated, one for each of the classes.

The TAN classifier is constructed based on the plans recovered that are most similar to the current plan, distinguishing between efficient and inefficient plans to generate the model (the tree). Thus, by applying the Friedman-Goldsmidtz [5] algorithm, the two classes that are considered are efficient and inefficient. The Friedman-Goldsmidtz algorithm makes it possible to calculate a Bayesian network based on the dependent relationships established through a metric. The metric considers the dependent relationships between the variables according to the classifying variable. In this case, the classified variable is efficient and the remaining variables indicate whether a service is or is not available. The metric proposed by Friedman can be defined as:

$$
I(X, Y \mid Z)=\sum_{x \in X} \sum_{y \in Y} \sum_{z \in Z} P(x, y, z) \cdot \log \left[\frac{P(x, y \mid z)}{P(x \mid z) \cdot P(y \mid z)}\right]
$$

Based on the previous metric, the probabilities are estimated according to the frequencies of the data. The Friedman-Goldsmidtz [23] algorithm is broken down into the following steps:

- Calculate the value of $I(X, Y \mid C)$ for the different variables/attributes $X, Y$ that may be interconnected in the original graph, class $C$ varies between the similar efficient and inefficient cases.

- Construct a complete nondirected graph

- Establish the different attributes/variables as nodes.

- Within the connections, establish the values obtained in the first step as weights. For the arcs that do not have connections, set the value as 0 .

- Create a maximum tree based on the Kruskal [6] algorithm.

- Convert the nondirected tree into a directed tree. The initial connection and the selection of the next node to connect will indicate the direction of the connections.

- Finally, construct the TAN model by adding a node that represents class $C$ and an arc that connects to $C$ for each of the attributes.

The revise phase includes statistical techniques to extract relevant variables during the classification process. There are a lot of variables therefore it is necessary and automatic method for extracting the relevant information for helping an expert during the reviewing process. The chi-square [12], the Yates correction tests [15], 
the chi-square with the Monte Carlo simulation [17], and Fisher's exact test [16] are applied to select the variables of interest that characterize the various pathologies. It is important to note that in order for the expected frequency to be less than 5 , the result may be incorrect; consequently Yates correction would be applied in an attempt to mitigate this issue. The statistical results from chi-squared are also provided, applying the Monte Carlo simulation to verify the results. Finally, an exact Fisher test is applied, which is the recommended method when the sample size is small and it is not possible to ensure that $80 \%$ of the data from a contingency table have a value greater than 5. Medical studies such as [14] use a process similar to the one presented for selecting variables that affect malformations; other biomedical studies include [15] [16] [17]. There are many alternatives for correcting data, such as that in [13]. The Figure 1 shows the CBR cycle and the techniques for each step.

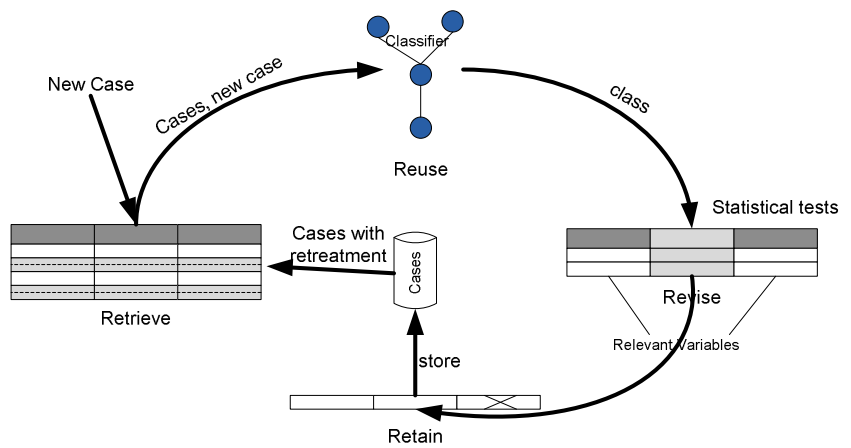

Fig 1 ROC curves with the classification accuracy.

\section{Results and Conclusions}

The selected cases were chosen from the patient files at the Faculty of Odontology, Masters of Endodontics, at the Complutense University of Madrid. All patients received root canal treatments between September 2000 and May 2011. Among all the patients treated during this time, we selected 35 cases that satisfied the inclusion criteria and were interested in a follow up appointment. None of patients from the selected cases who came for a follow up treatment refused to participate in the study.

A total of 18 women and 17 men were selected whose ages ranged from 18 to 85 years. The average age of the patients was 54.6 years and they all satisfied the inclusion critera as previously established. The selected cases contained all the information needed to complete the 72 variables being considered. These variables take into account all information relevant to the patient: medical and dental history, habits. Data relative to the state of the tooth prior to treatment were also included: the evolution, the clinical technique used, and the post treatment results. Certain initial variables included a high number of categories, which resulted in 
their recodification to ensure that the final number of categories per variable had around 3 or 4 different values. The stage that was most thoroughly analyzed during the study was the reuse stage. BayesNet, NaiveBayes, AdaBoostM1, Bagging, DecisionStump, J48, IBK, JRip, LMT, Logistic, LogitBoost, OneR, SMO and Stacking were analyzed. The following table shows the number of correct classifications obtained for each of the methods applying the leave one out technique to the CBR system, since the number of cases was not very high and cross validation could not therefore be applied. The rate of correct classifications for the system was $89 \%$.

Table 1 Correct classifications

\begin{tabular}{llll}
\hline Classifier & Correct & Classifier & Correct \\
\hline BayesNet & 88.57 & JRip & 60.00 \\
NaiveBayes & 82.86 & LMT & 65.71 \\
AdaBoostM1 & 68.57 & Logistic & 80.00 \\
Bagging & 68.57 & LogitBoost & 68.57 \\
DecisionStump & 42.86 & OneR & 65.71 \\
J48 & 77.14 & SMO & 77.14 \\
IBK & 82.86 & Stacking & 74.29 \\
\hline
\end{tabular}

The precision of the Bayes Net increased to 0.89 and recall to 1. Precision and recall are defined as follows.

$$
\text { precision }=t_{p} /\left(t_{p}+f_{p}\right) \text { recall }=t_{p} /\left(t_{p}+f_{n}\right)
$$

$t_{p}$ true positive, $f_{p}$ false positive, $f_{n}$ false negative.

A graphical representation with ROC curves was made with the previous results. The ROC curves facilitate the analysis of different classifiers according to the area represented beneath the curve. The bigger the area, the better the classifier. The main advantage is the ability to distinguish the relevance of false negatives compared to false positives. In this case, a positive is understood as a successful retreatment, given that the point is to avoid determining that an extraction is required if it were not actually necessary in the end. Figure 2 shows the ROC curve for each of the methods and the final result obtain. As shown, the result for the Bayesian network was satisfactory since the area beneath the curve is high and there are no false negatives (no extractions were predicted for successful cases).

To facilitate the revise phase, a revision was made to determine the difference between the values of the variables for the categories of successful retreatments and extractions. To perform this analysis, the Chi square, Yates correction, chi square with Monte Carlo simulation, and the Fisher's exact tests were applied. Table 2 displays the set of variables that were considered relevant by any of the three methods. We can see how the selection of variables coincides to a great degree for the different methods. 


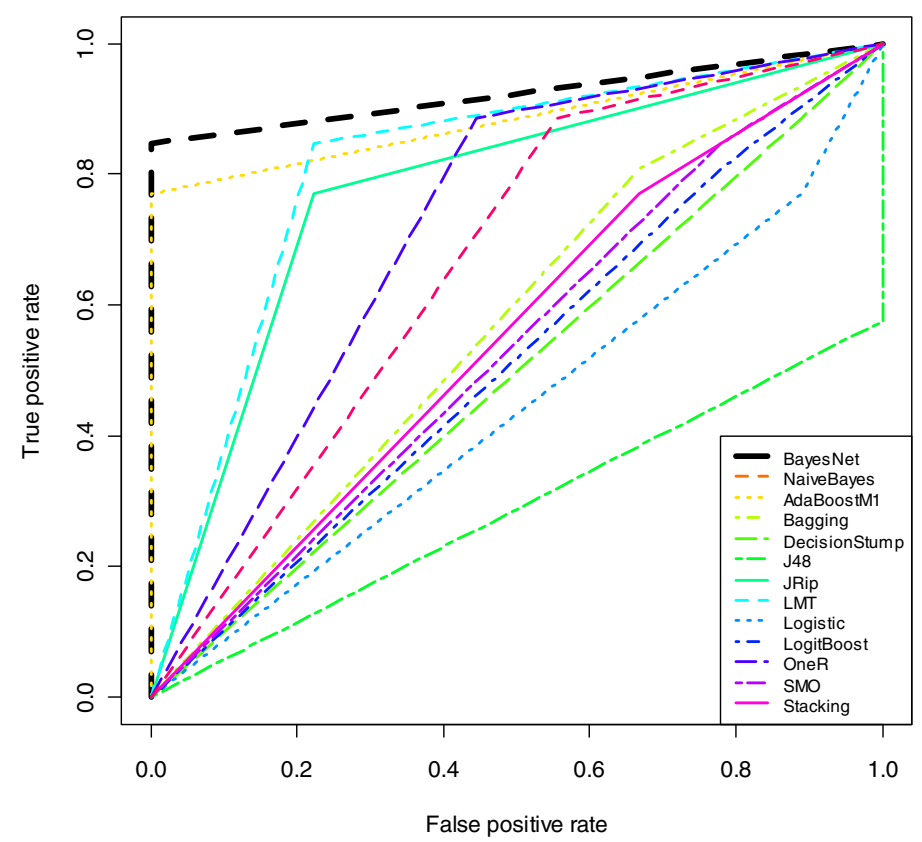

Fig 2 ROC curves with the classification accuracy

Table 2 Relevant variables

\begin{tabular}{|c|c|c|c|}
\hline \multirow{3}{*}{ Variable } & \multicolumn{3}{|c|}{ P value } \\
\hline & \multicolumn{2}{|c|}{ Chi-Squared } & \multirow{2}{*}{ Exact Fisher Test } \\
\hline & Yates & Monte Carlo & \\
\hline Reason for treatment & 0.007548049 & 0.004997501 & 0.008995502 \\
\hline Conde. Lateral or vertical & 0.073673116 & 0.037481259 & 0.044117647 \\
\hline Clamps & 0.052565842 & 0.052473763 & 0.033983008 \\
\hline Type of pain & 0.038628647 & 0.033983008 & 0.034482759 \\
\hline Cause of fracture & 0.00781095 & 0.008995502 & 0.002998501 \\
\hline Type of fracture & 0.005016858 & 0.00049975 & 0.001470355 \\
\hline Location & 0.022018021 & 0.013493253 & 0.018990505 \\
\hline Signs of fissure/fracture & 0.008699709 & 0.005997001 & 0.004272424 \\
\hline Probing & 0.005016858 & 0.001999 & 0.001470355 \\
\hline Visible fissure & 0.022879307 & 0.009995002 & 0.013070078 \\
\hline Level & 0.076170975 & 0.037481259 & 0.036300838 \\
\hline Other & 0.027888372 & 0.044977511 & 0.015492254 \\
\hline Retreatment & 0.000876579 & 0.00049975 & 0.000411132 \\
\hline
\end{tabular}


With the CBR analysis, the data obtained were relevant because by ordering the established variables, particularly those with the highest risk factor, we could predict the final solution for treatment and retreatment in $89 \%$ of the cases without obtaining any false negatives. Furthermore, the system makes it possible to extract the relevant variables that can distinguish the different types of individuals. Nevertheless, more cases are required to contrast the results with greater accuracy.

Acknowledgments. This work has been supported by the MICINN TIN 2009-13839-C03-03

\section{References}

1. Chugal, N.M., Clive, J.M., Spangberg, L.S.: A prognostic model for assessment of the outcome of endodontic treatment: Effect of biologic and diagnostic variables. Oral Surg. Oral Med. Oral Pathol. Oral Radiol. Endod. 91(3), 342-352 (2001)

2. Givol, N., et al.: Risk management in endodontics. J. Endod. 36(6), 982-984

3. Song, M., et al.: Prognostic factors for clinical outcomes in endodontic microsurgery: a retrospective study. J. Endod. 37(7), 927-933

4. Kolodner, J.: Case-Based Reasoning. Morgan Kaufmann (1993)

5. Friedman, N., Geiger, D., Goldszmidt, M.: Bayesian Network Classifiers. Machine Learning 29, 131-163 (1997)

6. Castro, J.L., Navarro, M., Sánchez, J.M., Zurita, J.M.: Loss and gain functions for CBR retrieval. Information Science 179(11), 1738-1750 (2009)

7. Joyanes, L., et al.: Knowledge Management. University of Paisley, Salamanca (2001)

8. Jurisica, I., Glasgow, J.: Applications of case-based reasoning in molecular biology. Artificial Intelligence Magazine 25(1), 85-95 (2004)

9. Canalda, C., Brau, E.: Endodoncia: técnicas clínicas y bases científicas, vol. 2. Masson, Barcelona (2006)

10. Casanellas, J.M.: Restauración del Diente Endodonciado, 1st edn. Pues, Madrid (2006)

11. Kruskal, W., Wallis, W.: Use of ranks in one-criterion variance analysis. Journal of American Statistics Association (1952)

12. Kenney, J.F., Keeping, E.S.: Mathematics of Statistics, Pt. 2, 2nd edn. Van Nostrand, Princeton (1951)

13. Martín Andrés, A., Silva Mato, A.: Optimal correction for continuity and conditions for validity in the unconditional chi-squared test. Computational Statistics \& Data Analysis 26(1), 609-626 (1996)

14. Himmetoglu, O., Tiras, M.B., Gursoy, R., Karabacak, O., Sahin, I., Onan, A.: The incidence of congenital malformations in a Turkish population. International Journal of Gynecology \& Obstetrics 55(2), 117-121 (1996)

15. Shaul, D.B., Scheer, B., Rokhsar, S., Jones, V.A., Chan, L.S., Boody, B.A., Malogolowkin, M.H., Mason, W.H.: Risk Factors for Early Infection of Central Venous Catheters in Pediatric Patients. Journal of the American College of Surgeons 186(6), 654-658 (1998)

16. Yang, X., Huang, Y., Crowson, M., Li, J., Maitland, M.L., Lussier, Y.A.: Kinase inhibition-related adverse events predicted from in vitro kinome and clinical trial data. 43(3), 376-384 (2010)

17. Nilsson, B.: A compression algorithm for pre-simulated MonteCarlop-value functions: Application to the ontological analysis of microarray studies. Pattern Recognition Letters 29(6), 768-772 (2008)

18. John, M., Priebe, C.E.: A data-adaptive methodology for finding an optimal weighted generalized Mann-Whitney-Wilcoxon statistic. Computational Statistics \& Data Analysis 51(9), 4337-4353 (2007) 